\section{OC-047 CORRELATION OF CAECAL INTUBATION RATE TO VOLUME - COLONOSCOPISTS SHOULD UNDERTAKE AT LEAST 120 PROCEDURES PER YEAR}

doi:10.1136/gutjnl-2013-304907.046

1."A M Verma, 'A Dixon, 'A Chilton. 'Gastroenterology, Kettering General Hospital NHS foundation trust, Kettering, UK

Introduction Quality assurance of colonoscopy is a central theme in endoscopic provision. In the UK the Joint Advisory Group on Gastrointestinal endoscopy (JAG), has issued guidance for caecal intubation rates (CIR) of $90 \%$ or higher on an intention-to-colonoscope basis. They also advise that practitioners should undertake at least 100 colonoscopies per annum. We looked at the correlation between volume per annum and CIR to test JAG's standard.

Methods We analysed 129 operator records who undertook between 20-399 colonoscopies per annum. This amounted to 12 594 colonoscopies undertaken over a two year period (2008-9) across 6 hospitals in 3 regions. Each operator's volume per annum was plotted against CIR. This was done as individual operators and also when individuals were grouped with others performing a similar volume of colonoscopy per annum (groups of 50). An additional analysis of operators undertaking 20-199 colonoscopies per annum looked at groups of 20 and groups of 10 .

Results Figure A shows a scatter plot of individuals' volume (per annum) against CIR, the trend-line crosses the $90 \%$ CIR standard at 125 procedures per annum. The 3 other scatter plots of individuals grouped with others undertaking a similar volume per annum (groups of 50, 20 and 10) plotted against CIR, the trendline crosses the $90 \%$ CIR standard at 110-120 procedures per annum.

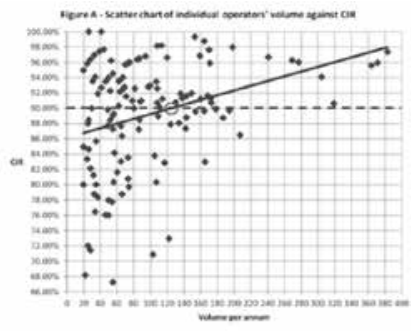

\section{Abstract 0C-047 Figure}

Conclusion The analysis of 12594 colonoscopies, performed by 129 operators, carrying out between 20-399 colonoscopies per annum, suggest that to meet a minimum CIR standard of $90 \%$ at least 120 colonoscopies should be performed per annum. This is equivalent to performing 3 colonoscopies per week. We recommend advisory bodies on quality standards adopt this minimum activity threshold into their guidance.

Disclosure of Interest None Declared

\section{BSG trainees symposium: making your training work for you}

\section{OC-048 COLONOSCOPY: WHAT IS THE NUMBER REQUIRED TO MAINTAIN COMPETENCY? - A RETROSPECTIVE AUDIT}

doi:10.1136/gutjnl-2013-304907.047

1." C Kong, ${ }^{1} \mathrm{~A} N \mathrm{~N}$ Young, ${ }^{1} \mathrm{~K}$ Benson, ${ }^{1} \mathrm{~J} \mathrm{~J}$ Keating, ${ }^{1} \mathrm{~A}$ H Davies. ${ }^{1}$ Gastroenterology, Furness General Hospital, University Hospital Morecombe Bay Trust, Barrow-in-Furness, UK

Introduction There has been increasing demand for gastroenterologist to maintain JAG competencies year on year. ${ }^{1}$ However there are few studies into the adequate number required despite the bowel cancer screening programme suggesting $>100$ per annum. The study aims to compare competencies standards in relation to the number of colonoscopies performed in a year.

Methods Data was collected retrospectively within the trust between 1/12/2010 and 30/11/2011. These include colonoscopies performed in the 3 main hospitals within the UHMBT - Royal Lancaster Infirmary, Furness General Hospital and Westmoreland General Hospital. A total of 2502 colonoscopies performed were retrieved. The endoscopy reporting system 'Endobase' and 'Indigo 4 Review' were used to retrieve endoscopy report and pathology report respectively. The caecal intubation rate (completion rate), polyp detection rate, adenoma detection rate, adequacy of bowel prep and sedation rate were set standards. Chi squared test for correlation was used to compare all standards. Two groups were formed to compare the set standards as colonoscopist who performed $>100$ colonoscopies per annum (pa) and those who performed $<100$ colonoscopies pa. Withdrawal time, polyp recovery and complication rates were not included in this audit

Results Of the 2502 colonoscopies performed, 633 were performed by colonoscopist who performed $<100,1869$ colonoscopies were performed by colonoscopist who performed $>100$ colonoscopies. Completion rate for $<100$ colonscopies was $85.62 \%$ and $>100$ colonoscopies was $92.24 \%(p<0.0001)$. Polyp detection rate (PDR) for $<100$ colonscopies was $17.85 \%$ and $>100$ colonoscopies was $30.34 \%$ ( $p<0.0001)$. Adenoma detection rate for $<100$ colonoscopies was $25.28 \%$ and $>100$ colonoscopies was $32.98 \%(p=0.0003)$. Comparison of completion rates between both good and satisfactory bowel prep was $91.27 \%$ against poor prep $81.25 \%(p<0.001)$ Reasons for non completion in order of frequency were patient discomfort, excess looping, pathology encountered limiting progression, inadequate bowel preparation, tight stricture and instrument inadequacy. For sedation, the number of times pethidine given $>$ $50 \mathrm{mg}$ was $0.15 \%$ and number of times midazolam given $>5 \mathrm{mg}$ was $0.05 \%$

Conclusion Performing at least 100 colonoscopies a year statistically improves completion rate, polyp detection rate and adenoma detection rate. Good and satisfactory bowel prep statistically improves completion rate. This audit supports the JAG recommendation that a colonoscopist should perform $>100$ colonoscopies per annum to maintain competency.

Disclosure of Interest None Declared

\section{REFERENCE}

1. Valori R, Barton R. BSG quality and safety indicators for endoscopy. JAG: Joint Advisory Group on GI Endoscopy. 2007; 1-13.

\section{OC-049 ARE ENDOSCOPIC EXAMINATIONS PERFORMED BY TRAINEE ENDOSCOPISTS LESS TOLERABLE THAN THOSE CONDUCTED BY SENIOR COLLEAGUES?}

doi:10.1136/gutjnl-2013-304907.048

${ }^{1}$ A J Irvine, 1."M Kurien, 'R Harrold, 'L Taylor, 'D S Sanders. 'Department of Gastroenterology, Royal Hallamshire Hospital, Sheffield, UK

Introduction Endoscopic training for gastroenterology trainees is an integral part of specialty training, with defined competencies required prior to independent practise. Whilst training of future endoscopists is essential to meet the future demands of endoscopy, previous studies have suggested that tolerability and patient satisfaction is decreased when trainees undertake endoscopic examinations. This study evaluates the influence trainees may have on endoscopic tolerability with 3 outcome measures assessed: procedural pain, discomfort and distress. 